
лечения рака прямой кишки и ректосигмоидного соединения

\author{
Иванов Ю.В. ${ }^{1,2}$ • Смирнов А.В. ${ }^{1}$ Винокуров А.В. ${ }^{1}$ З Злобин А.И. ${ }^{1}$ Станкевич В.Р. $~ •$ \\ Данилина Е.C.
}

Иванов Юрий Викторович - д-р мед. наук, профессор, заведующий хирургическим отделением', гл. науч. сотр. лаборатории минимально инвазивной хирургии²; ORCID: https://orcid.org/0000-0001-62094194. E-mail: ivanovkb83@yandex.ru

Смирнов Александр Вячеславович - канд. мед. наук, врач-хирург хирургического отделения';

ORCID: https://orcid.org/0000-0003-3897-8306

$\triangle 115682$, г. Москва, Ореховый бульвар, 28,

Российская Федерация. Тел.: +7 (926) 2048148.

E-mail: alvsmirnov@mail.ru

Винокуров Александр Витальевич - ординатор кафедры хирургии Академии постдипломного образования'; ORCID: https://orcid.org/0000-0001-80261216. E-mail: vinokuroff.sasha2015@yandex.ru

Злобин Александр Иванович - канд. мед. наук, врачхирург хирургического отделения'

Станкевич Владимир Романович - врач-хирург хирургического отделения'; ORCID: https://orcid. org/0000-0002-8620-8755.

E-mail: v-stankevich@yandex.ru

Данилина Екатерина Станиславовна - врач-хирург хирургического отделения, старший лаборант кафедры хирургии Академии постдипломного образования'; ORCID: https://orcid.org/0000-0002-24663795. E-mail: danilina.katja@bk.ru

\begin{abstract}
Цель - оценить эффективность комбинации механической очистки кишечника (МОК) с пероральным приемом антибактериальных препаратов при подготовке пациента к передней резекции прямой кишки в профилактике послеоперационных осложнений.

Материал и методы. Проанализированы ближайшие результаты лечения 77 пациентов, которым выполнена передняя резекция прямой кишки по поводу рака прямой кишки и рака ректосигмоидного соединения. 45 пациентов были подготовлены к операции только в объеме МОК. У 32 пациентов перед операцией помимо МОК были назначены пероральные антибактериальные препараты ципрофлоксацин и метронидазол.

Результаты. Общий уровень послеоперационных осложнений составил 6,25\% (2 из 32 пациентов) в группе комбинированной подготовки к операции и 15,5\% (7 из 45 пациентов) в группе применения только МОК. Нагноение раны развилось у 1 пациента в группе комбинированной подготовки и у 4 больных в группе применения только МОК. Несостоятельности анастомоза в группе комбинированной подготовки не было, тогда как при использовании только МОК несостоятельность анастомоза отмечена у 2 больных.
\end{abstract}

Заключение. Использование комбинации приема пероральных антибактериальных препаратов в сочетании с МОК перед передней резекцией прямой кишки позволяет достичь крайне низкой частоты несостоятельности колоректального анастомоза. Необходимы дальнейшие исследования эффективности данной схемы подготовки с их обсуждением на уровне профессиональных сообществ.

Ключевые слова: колоректальный рак, передняя резекция прямой кишки, кишечная микрофлора, несостоятельность колоректального анастомоза

Для цитирования: Иванов ЮВ, Смирнов $A B$, Винокуров АВ, Злобин АИ, Станкевич ВР, Данилина ЕС. Предоперационная механическая очистка кишечника в сочетании с пероральным приемом антибактериальных препаратов в профилактике осложнений хирургического лечения рака прямой кишки и ректосигмоидного соединения. Альманах клинической медицины. 2021;49(7):477484. doi: 10.18786/2072-0505-2021-49-062.

Поступила 06.09.2021; доработана 11.12.2021; принята к публикации 13.12.2021; опубликована онлайн 16.12.2021

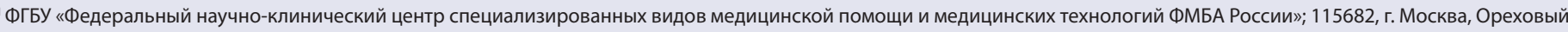
6-р, 28, Российская Федерация

2 ФГБОУ ВО «Московский государственный медико-стоматологический университет имени А.И. Евдокимова» Минздрава России; 127473, г. Москва, ул. Делегатская, 20-1, Российская Федерация

$\mathrm{P}$ ак прямой кишки и ректосигмоидного соединения входит в десятку самых распространенных в России злокачественных новообразований с численностью контингента больных 118 человек на 100000 населения. В 2019 г. зарегистрировано 30244 новых случая заболевания. При этом ведущим методом лечения остается хирургический: в 2019 г. было оперировано 93,3\% пациентов, подлежащих радикальному лечению [1]. Наиболее грозным осложнением передней резекции прямой кишки признается несостоятельность колоректального анастомоза, которая возникает у $1,5-15,2 \%$ больных, а летальность при этом достигает 4,7\% [2-4]. 
Полагают, что подобно заживлению кожной раны процесс заживления колоректального анастомоза проходит через 3 фазы: воспалительную, пролиферативную и ремоделирования. В ходе воспалительной фазы анастомоз стремительно теряет свою прочность, которая уменьшается на 70\% в течение ближайших 48 часов [5]. В дальнейшем фибробласты и гладкомышечные клетки синтезируют новый коллаген, который укрепляет анастомоз. Нарушение процесса заживления связано с развитием несостоятельности анастомоза.

По всей вероятности, важную роль в заживлении анастомоза играет микрофлора кишечника. Выделяют 2 вида кишечной микробиоты с разным составом: микробиоту просвета кишечника и слизистой кишки. Показано, что при высокой численности Bacteroidaceae и Lachnospiraceae воспалительная фаза затягивается, а бактериальные коллагеназы вызывают истощение кишечного коллагена [6].

В последние годы появились данные, согласно которым оптимальный состав микрофлоры достигается при подготовке пациента к операции путем сочетания механической очистки кишечника (МОК) и приема пероральных антибактериальных препаратов (ППАП). МОК уменьшает объем фекалий и улучшает доставку антибактериальных препаратов к слизистой оболочке кишечника [6]. Данная методика потенциально должна приводить к уменьшению частоты несостоятельности колоректального анастомоза в послеоперационном периоде, однако в ходе ранее проведенных исследований были получены противоречивые результаты, что и обусловливает актуальность настоящей работы.

Цель исследования - оценить эффективность сочетания приема ППАП с МОК при подготовке пациента к передней резекции прямой кишки в профилактике послеоперационных осложнений.

\section{Материал и методы}

Проведено наблюдательное ретроспективное сплошное исследование. В 2017-2020 гг. в Федеральном научно-клиническом центре специализированных видов медицинской помощи и медицинских технологий ФМБА России авторами выполнено 77 передних резекций прямой кишки по поводу рака прямой кишки (60 пациентов) или ректосигмоидного соединения (17 пациентов). Характеристики пациентов отражены в табл. 1. Мужчин было 40, женщин - 37. Медианный возраст составил 65 лет, интерквартильный размах - от 60 лет до 71 года.
В исследование включены все пациенты, оперированные в центре за 4-летний период. Начиная с сентября 2018 г. всем пациентам проводили комбинированную подготовку в виде МОК в сочетании с ППАП, до этого момента применяли только MOK.

Проанализированы ближайшие результаты хирургического лечения, сравнение проводили между двумя группами пациентов:

1) пациенты, которым в качестве предоперационной подготовки выполнена МОК в комбинации с ППАП (с сентября 2018 по декабрь 2020 г., 32 больных);

2) пациенты, которым в качестве предоперационной подготовки выполнялась только МОК (с января 2017 по август 2018 г. набрано 45 больных).

В целом группы были однородными по изученным характеристикам. В группе комбинированной подготовки превалировало высокое расположение опухоли, тогда как в группе использования МОК чаще регистрировали среднюю локализацию. Вместе с тем пациентов с низким расположением опухоли, для которых риск развития осложнений традиционно считается выше, в процентном соотношении в группе комбинированной подготовки было даже больше.

Механическую очистку кишечника проводили с применением слабительных средств на основе макрогола в соответствии с инструкцией по медицинскому использованию данных препаратов. Пероральная антибактериальная профилактика включала прием ципрофлоксацина 500 мг в сочетании с метронидазолом 2 г в 19:00 и 22:00.

3а 30-40 минут до операционного разреза начинали парентеральную антибиотикопрофилактику ципрофлоксацином 400 мг и метронидазолом 500 мг внутривенно. Парентеральную антибиотикопрофилактику не продолжали более 24 часов.

Лапароскопическим доступом оперировано 53 пациента, открытым - 24.

Техника операции. У всех пациентов выполняли переднюю резекцию прямой кишки с тотальной или субтотальной мезоректумэктомией. При локализации опухоли выше уровня тазовой брюшины проводили высокое пересечение нижней брыжеечной артерии в области ее устья, при расположении опухоли ниже уровня тазовой брюшины нижнюю брыжеечную артерию пересекали сразу после отхождения левой ободочной артерии, но с обязательным удалением апикальных лимфоузлов. Прямую кишку пересекали артикуляционным эндоскопическим сшивающе-режущим 
Таблица 1. Основные характеристики больных

\begin{tabular}{|c|c|c|c|}
\hline \multirow[t]{2}{*}{ Показатель } & \multicolumn{2}{|l|}{ Тип операции } & \multirow[t]{2}{*}{ Значение $p$} \\
\hline & MOK + ППАП (n=32) & MOK $(n=45)$ & \\
\hline Возраст, Ме [Q1; Q3], годы & $64[60 ; 69]$ & $66[59 ; 76]$ & 0,32 \\
\hline Мужчины, абс. (\%) & $15(46,9)$ & $25(55,5)$ & 0,45 \\
\hline Женщины, абс. (\%) & $17(53,1)$ & $20(44,5)$ & 0,45 \\
\hline Рак прямой кишки, абс. (\%) & $23(71,9)$ & $37(82,2)$ & 0,28 \\
\hline Рак ректосигмоидного соединения, абс. (\%) & $9(28,1)$ & $8(17,8)$ & 0,28 \\
\hline ASA II, абc. (\%) & $8(25)$ & $15(33,3)$ & 0,43 \\
\hline ASA III, абс. (\%) & $18(56,3)$ & $22(48,9)$ & 0,52 \\
\hline ASA IV, абс. (\%) & $6(18,7)$ & $8(17,8)$ & 0,91 \\
\hline \multicolumn{4}{|l|}{ Локализация опухоли (расстояние до ануса), абс. (\%): } \\
\hline низкая (от 3 до 5 см) & $9(28,1)$ & $8(17,8)$ & 0,218 \\
\hline средняя (от 6 до 10 см) & $8(25)$ & $24(53,3)$ & 0,01 \\
\hline высокая (свыше 11 см) & $15(46,9)$ & $13(28,9)$ & 0,06 \\
\hline Предшествующая химиолучевая терапия, абс. (\%) & $5(15,6)$ & $5(11,1)$ & 0,56 \\
\hline Лапароскопический доступ, абс. (\%) & $20(62,5)$ & $33(73,3)$ & 0,31 \\
\hline Превентивная трансверзостома, абс. (\%) & $13(40,6)$ & $15(33,3)$ & 0,51 \\
\hline
\end{tabular}

ASA - классификация объективного статуса больного, разработанная Американским обществом анестезиологов (ASA); MOK - механическая очистка кишечника;

ППАП - прием пероральных антибактериальных препаратов

аппаратом. Колоректальный анастомоз «конец в конец» формировали с помощью циркулярного сшивающего аппарата с диаметром головки от 28 до 31 мм. Во всех случаях мы применяли следующую технику наложения аппаратного анастомоза. После введения аппарата в культю прямой кишки выдвижение троакара через стенку кишки осуществляли ближе к левому углу культи. Таким образом, после прошивания и пересечения ткани кишки оставался только один угол (правый) анастомозированной культи прямой кишки, а не два, как при стандартной методике наложения аппаратного шва. Оставшийся угол погружали в просвет кишки с помощью одного П-образного шва, надежно закрывая тем самым участок углового скрепочного шва. Во всех случаях проводили интраоперационную оценку герметичности анастомоза с помощью воздушной пробы. Если проба была положительной или сомнительной, накладывали дополнительные серозно-мышечные швы атравматической иглой на 2, 4, 6, 8, 10 и 12 часах по условному циферблату. При низком расположении колоректального анастомоза (менее 10 см от наружного края анального канала), а также при отягчающих факторах его формирования или наличии сопутствующих заболеваний, увеличивающих риск его несостоятельности, дополнительно накладывали превентивную разгрузочную двухствольную трансверзостому в эпигастрии или левом подреберье. Во всех случаях операцию заканчивали или чрезбрюшинным дренированием малого таза, или чреспромежностным пресакральным дренированием крестцовой ямки.

В послеоперационном периоде оценивали клинические и лабораторные показатели, при необходимости выполняли рентгенологические и ультразвуковые исследования. В случае выявления несостоятельности колоректального анастомоза для оценки ее тяжести применяли классификацию International Study Group of Rectal Cancer (A - необходима активная терапия; В - необходима активная консервативная терапия, но без релапаротомии; C - необходима релапаротомия) 
[3]. Дальнейшая тактика лечения зависела от клинико-лабораторных данных и степени тяжести возникшего осложнения.

Сравнительной оценке подвергнуты лишь непосредственные результаты оперативного лечения в 2 группах: послеоперационные осложнения, послеоперационный койко-день, послеоперационная летальность и отдельно количество несостоятельностей колоректального анастомоза.

Изучено только влияние предоперационной подготовки на ближайший результат операции. Поскольку мы считаем группы однородными, отдельное воздействие таких факторов, как локализация опухоли, предшествующая лучевая терапия, вид операционного доступа, не учитывали.

Статистический анализ полученных результатов проводили с использованием непараметрических методов. Данные представлены в виде медианы с указанием интерквартильного размаха. Различия между количественными характеристиками определяли с помощью критерия Манна - Уитни. Сравнение качественных характеристик проводили при помощи метода $\chi^{2}$ (хи-квадрат). Полученные различия признавались статистически значимыми при двустороннем $\mathrm{p}<0,05$ (95\% точности).

\section{Результаты}

Характеристика непосредственных результатов оперативного лечения отражена в табл. 2. Общий уровень послеоперационных осложнений составил $6,25 \%$ в группе комбинированной подготовки к операции и 15,5\% в группе применения только МОК. Нагноение раны развилось у 1 пациента в группе комбинированной подготовки и у 4 больных в группе использования только МОК, при этом у одного из них была эвентрация, потребовавшая устранения в условиях операционной под общим обезболиванием. У 2 пациентов (по одному в каждой группе) в течение первых суток послеоперационного периода развилось внутрибрюшное кровотечение, потребовавшее хирургического гемостаза.

Несостоятельность колоректального анастомоза возникла только в 2 наблюдениях в группе МОК, в группе комбинированной подготовки несостоятельностей анастомоза не было. В одном наблюдении анастомоз располагался на расстоянии 5 см от наружного края анального канала, в другом - 6 см. В обоих случаях первичные операции у данных пациентов были закончены формированием превентивной трансверзостомы. У одного больного из сопутствующих заболеваний имелся сахарный диабет 2-го типа (уровень гликированного гемоглобина 8,5\%) на фоне ожирения II степени (индекс массы тела 37), другой пациент перед оперативным лечением проходил курсы неоадъювантной химиотерапии. Согласно классификации International Study Group of Rectal Cancer, возникшие несостоятельности колоректального анастомоза отнесены к классам А и В, то есть повторного оперативного вмешательства не требовалось. При этом лишь в 1 случае

Таблица 2. Результаты оперативного лечения

\begin{tabular}{|c|c|c|c|}
\hline \multirow[t]{2}{*}{ Характеристика пациентов } & \multicolumn{2}{|l|}{ Тип операции } & \multirow[t]{2}{*}{ Значение $p$} \\
\hline & MOK + ППАП $(n=32)$ & $\operatorname{MOK}(n=45)$ & \\
\hline Продолжительность операции, Me [Q1; Q3], часы & $180[150 ; 210]$ & $205[180 ; 277]$ & 0,11 \\
\hline Кровопотеря, Мe [Q1; Q3], мл & $100[100 ; 250]$ & $100[100 ; 212]$ & 0,92 \\
\hline \multicolumn{4}{|l|}{ Осложнения по Clavien-Dindo, абс. (\%) } \\
\hline$|-| \mid$ & $1(3,12)$ & $5(12,5)$ & 0,198 \\
\hline III-V & $1(3,12)$ & $2(4,4)$ & 0,77 \\
\hline \multicolumn{4}{|c|}{ Несостоятельность колоректального анастомоза по ISGRC, абс. (\%) } \\
\hline A & 0 & $1(2,2)$ & 0,39 \\
\hline B & 0 & $1(2,2)$ & 0,39 \\
\hline Послеоперационный койко-день, Ме [Q1; Q3] & $8[7 ; 10]$ & $9[7 ; 13]$ & 0,9 \\
\hline Летальность, абс. & 0 & 0 & \\
\hline
\end{tabular}

ISGRC - классификация International Study Group of Rectal Cancer, MOK - механическая очистка кишечника, ППАП - прием пероральных антибактериальных препаратов 
на 3-и сутки после операции наблюдали клинико-лабораторную картину осложнения (класс В), проявившуюся субфебрильной температурой тела $\left(37,6^{\circ} \mathrm{C}\right)$, повышением уровня лейкоцитов в крови до $15,4 \times 10^{9} /$ л, С-реактивного белка до 70 мг/л, a также появлением незначительного количества (30 мл) мутного отделяемого по страховочному дренажу, выведенному через переднюю брюшную стенку. Отсутствие симптомов раздражения брюшины, признаков свободной жидкости в брюшной полости и малом тазу по данным ультразвукового исследования, наличие функционирующей превентивной трансверзостомы позволили избрать консервативную тактику ведения больного. На фоне скорректированной антибактериальной терапии (цефоперазон + сульбактам по 4,0 г 2 раза в сутки внутривенно капельно + линезолид по 600 мг 2 раза в сутки внутривенно капельно) и ежедневного двукратного промывания страховочного дренажа (0,9\% раствор натрия хлорида) состояние пациента стабилизировалось, лабораторные показатели нормализовались, выписан на 11-е сутки после операции в удовлетворительном состоянии.

У второго больного несостоятельность колоректального анастомоза возникла на 4-е сутки после операции и была отнесена к классу А, так как отсутствовали какие-либо клинико-лабораторные проявления. Осложнение диагностировано по характеру отделяемого по страховочному дренажу, установленному чреспромежностно. Как и в первом случае, несостоятельность колоректального анастомоза подтверждена с помощью проктографии. Данное осложнение не повлияло на проводимую послеоперационную терапию, и пациент был выписан на 7-е сутки после операции в удовлетворительном состоянии.

\section{Обсуждение}

Механическая очистка кишечника перед колоректальными операциями оставалась догмой на протяжении более века. Однако в значительном числе исследований не удалось доказать каких-либо преимуществ МОК перед отсутствием какой-либо подготовки в принципе. Вместе с тем недостатки методики хорошо известны: дискомфорт пациента, нарушения водно-электролитного баланса, изменения кишечной микрофлоры и слизистой толстой кишки с увеличением эффекта бактериальной транслокации $[7,8]$. От применения МОК постепенно отказывались после накопления результатов об ее неэффективности в многочисленных рандомизированных контролируемых исследованиях [9, 10], кульминацией которых стала публикация в 2011 г. Кокрановского систематического обзора, подтвердившего отсутствие преимуществ использования МОК [11]. Более того, в другом метаанализе установлено, что МОК фактически увеличивает риск послеоперационной хирургической инфекции [12].

Высокая эффективность пероральных антибактериальных препаратов в сочетании с MOK обнаружена фактически случайно [13]. В двух крупных ретроспективных исследованиях, основанных на данных Национальной программы улучшения качества хирургической помощи Американского колледжа хирургов (ASC-NSQIP), представлены данные о том, что MOK в комбинации с пероральными антибактериальными препаратами сокращает послеоперационные осложнения почти вдвое $[14,15]$.

В 2018 г. K.E. Rollins и соавт. провели всесторонний систематический обзор и метаанализ рандомизированных клинических исследований и обсервационных когортных исследований с целью сравнения связи между послеоперационными результатами в колоректальной хирургии при использовании пероральных антибактериальных препаратов с МОК или без МОК [16]. Комбинация МОК с пероральными антибактериальными препаратами по сравнению с одной МОК ассоциировалась со значительным снижением хирургической инфекции (отношение рисков (ОР) 0,51), несостоятельности анастомоза (ОР 0,62), 30-дневной летальности (ОР 0,58), количества послеоперационных осложнений (ОР 0,67), кишечной непроходимости $(\mathrm{OP} 0,72)$ без разницы в частоте инфицирования Clostridium difficile [16].

В 2018 г. J.W.T. Toh и соавт. представили результаты метаанализа рандомизированных клинических исследований, посвященных влиянию различных режимов подготовки кишечника на хирургические инфекционные осложнения [17]. Их окончательные результаты показали значительное снижение риска общих и послеоперационных инфекционных осложнений при использовании МОК в комбинации с пероральными антибактериальными препаратами. Данный режим подготовки к операции был признан авторами лучшим [17].

В 2017 г. группой Европейского общества колопроктологии было проведено международное многоцентровое проспективное исследование пациентов, перенесших левостороннюю резекцию толстой кишки. Оно было направлено на установление связи между режимом подготовки пациента и риском развития несостоятельности анастомоза [18]. Из 3676 пациентов 618 получали перед операцией МОК и пероральные 
антибактериальные препараты. Эта группа имела самый низкий уровень несостоятельностей $(6,1 \%)$ по сравнению с пациентами, получавшими только МОК (9,2\%), и пациентами, не получившими никакой подготовки (8,7\%) [18].

В 2019 г. опубликованы результаты многоцентрового рандомизированного клинического исследования SELECT, в котором изучали роль селективной деконтаминации желудочно-кишечного тракта в снижении частоты несостоятельностей анастомозов у пациентов, перенесших колоректальную операцию [19]. В группе применения селективной деконтаминации желудочно-кишечного тракта в сравнении с группой приема только цефазолина в сочетании с метронидазолом отмечено значительное снижение послеоперационных инфекционных осложнений (14,9 против 26,9\%). Однако в частоте развития несостоятельностей анастомозов достоверной разницы получено не было, хотя их количество было меньше в группе селективной деконтаминации желудочно-кишечного тракта (6,1 против 9,7\%).

В 2020 г. были опубликованы результаты рандомизированного клинического исследования, проведенного в 1999-2004 гг. [20]. Эффективность МОК + ППАП по сравнению с МОК изучали у пациентов, перенесших резекцию прямой кишки. Исследование было остановлено при промежуточном анализе после набора только 80 пациентов (первоначально запланировано 280 пациентов) из-за статистически значимого снижения риска несостоятельности анастомоза у пациентов, получавших МОК + ППАП (5\% против $20 \%, \mathrm{p}=0,0425)$.

Не во всех исследованиях комбинация МОК в сочетании с ППАП показала хороший эффект. В многоцентровом рандомизированном клиническом исследовании MOBILE (2019) оценивали влияние использования МОК и пероральных антибактериальных препаратов в сравнении с отсутствием подготовки кишечника на снижение риска хирургических инфекционных осложнений. В исследование включались все колоректальные операции, резекции прямой кишки составляли 2,5\% исследуемой популяции. Результаты не показали какой-либо существенной разницы в отношении раневых инфекционных осложнений, несостоятельности анастомоза или частоты повторных операций между двумя группами [21].

R. Garfinkle и соавт. проанализированы результаты колоректальных операций у 40446 пациентов [22]. МОК снижала вероятность развития несостоятельности анастомоза по сравнению с отсутствием какой-либо подготовки на 16\%, ППАП - на 40\%, комбинация МОК с ППАП - на 48\%. Статистически значимых различий при сравнении ППАП с комбинацией МОК и ППАП Получено не было [22].

В настоящее время ведущие профессиональные сообщества США закрепили в своих рекомендациях комбинацию МОК с ППАП в качестве ведущего способа подготовки пациента к хирургическому вмешательству [23-25]. Однако на общемировом уровне признания данного способа подготовки как единственно правильного пока нет. Еще более важно, что такая комбинированная подготовка повсеместно не закреплена на локальном уровне, в хирургических стационарах, где осуществляются колоректальные операции.

В нашем исследовании статистически значимых различий по количеству послеоперационных осложнений и несостоятельностей анастомоза мы не получили, несмотря на отчетливую тенденцию к более благоприятному течению послеоперационного периода у пациентов с комбинированной подготовкой (МОК+ППАП). Данный факт связан с тем, что при общем небольшом количестве осложнений для получения статистической значимости необходимо исследование большего числа пациентов.

Наше исследование имеет и другие ограничения: оно носит ретроспективный характер; отсутствует рандомизация; однородность сравниваемых групп достаточна условна, поскольку в связи с малым количеством общей выборки мы не имели возможность отдельно учесть влияние локализации и распространенности опухоли, предшествующей лучевой терапии, оперативного доступа, индекса массы тела, наличия и степени выраженности сахарного диабета, приема стероидных препаратов, курения, гипоальбуминемии, степени потери массы тела и др.

\section{Заключение}

Сегодня имеются доказательства эффективности МОК в сочетании с ППАП в профилактике послеоперационных осложнений при резекции прямой кишки. Использование данной методики в комбинации с рядом технических особенностей выполнения операции позволяет достичь крайне низкой частоты несостоятельностей колоректального анастомоза. Однако отсутствие крупных проспективных рандомизированных исследований не позволяет закрепить данный способ предоперационной подготовки в качестве ведущего на мировом уровне. Необходимы дальнейшие исследования эффективности комбинированной подготовки пациента с применением МОК и ППАП перед передней резекцией прямой кишки с их обсуждением на уровне профессиональных сообществ. (๕) 


\section{Дополнительная информация}

\section{Финансирование}

Работа проведена в рамках выполнения Государственного задания Федерального медико-биологического агентства № 121031900022-7.

Конфликт интересов

Авторы заявляют об отсутствии конфликта интересов.

Участие авторов

Ю.В. Иванов - концепция и дизайн исследования, написание и редактирование текста; А.В. Смирнов - концепция и дизайн исследования, анализ данных, написание и редактирование текста; А.В. Винокуров, B.P. Станкевич и Е.С. Данилина - сбор и обработка материала; А.И. Злобин - статистическая обработка данных, подбор и анализ литературы. Все авторы прочли и одобрили финальную версию статьи перед публикацией, согласны нести ответственность за все аспекты работы и гарантируют, что ими надлежащим образом были рассмотрены и решены вопросы, связанные с точностью и добросовестностью всех частей работы.

\section{Литература / References}

1. Каприн АД, Старинский ВB, Шахзадова АО, ред. Состояние онкологической помощи населению России в 2019 году. М.: МНИОИ им. П.А. Герцена - филиал ФГБУ «НМИЦ радиологии» Минздрава России; 2020. 239 с. [Kaprin AD, Starinskiy VV, Shakhzadova AO, editors. The state of cancer care to the population of Russia in 2019. Moscow: P. Hertsen Moscow Oncology Research Institute - Branch of the National Medical Research Radiological Centre of the Ministry of Health of the Russian Federation; 2020. 239 p. Russian.]

2. Кит ОИ, Геворкян ЮА, Солдаткина НВ. Пути улучшения результатов применения аппаратного анастомоза в хирургии рака прямой кишки. Хирургия. Журнал им. Н.И. Пирогова. 2013;(12):37-42. [Kit OI, Gevorkian luA, Soldatkina NV. [Ways to improve the results of the staple suture use for the rectal anastomosis]. Khirurgiya. 2013;(12):37-42. Russian.]

3. Алексеев МВ, Шелыгин ЮА, Рыбаков ЕГ. Трансанальное укрепление низкого колоректального анастомоза: первый опыт и перспективы. Колопроктология. 2016;(4): 15-21. doi: 10.33878/2073-7556-2016-0-4-1521. [Alekseev MV, Shelygin YuA, Rybakov EG. [The transanal reinforcement of low colorectal anastomosis: first experience and perspectives]. Koloproktologia [Coloproctology]. 2016;(4):15-21. Russian. doi: 10.33878/20737556-2016-0-4-15-21.]

4. Попов ДЕ. Факторы риска несостоятельности колоректальных анастомозов у больных раком прямой кишки. Колопроктология. 2014;(2):48-56. [Popov DE. [Risk factors of anastomotic leakage in patients with rectal carcinoma]. Koloproktologia [Coloproctology]. 2014;(2):48-56. Russian.]

5. Guyton KL, Hyman NH, Alverdy JC. Prevention of perioperative anastomotic healing complications: Anastomotic stricture and anastomotic leak. Adv Surg. 2016;50(1):129-141. doi: 10.1016/j.yasu.2016.03.011.

6. Agnes A, Puccioni C, D'Ugo D, Gasbarrini A, Biondi A, Persiani R. The gut microbiota and colorectal surgery outcomes: facts or hype? A narrative review. BMC Surg. 2021;21(1):83. doi: 10.1186/s12893-021-01087-5.
7. Jung $B$, Lannerstad $O$, Påhlman L, Arodell $M$, Unosson $M$, Nilsson E. Preoperative mechanical preparation of the colon: the patient's experience. BMC Surg. 2007;7:5. doi: 10.1186/1471-2482-7-5.

8. Bucher P, Gervaz P, Egger JF, Soravia C, Morel P. Morphologic alterations associated with mechanical bowel preparation before elective colorectal surgery: a randomized trial. Dis CoIon Rectum. 2006;49(1):109-112. doi: 10.1007/ s10350-005-0215-5.

9. Goldstone AR, Kennedy N, Metcalfe M. Randomized clinical trial of mechanical bowel preparation versus no preparation before elective left-sided colorectal surgery ( $\mathrm{Br} J$ Surg 2004; 92: 409-414). Br J Surg. 2005;92(8):1046. doi: 10.1002/bjs.5127.

10.Zmora O, Mahajna A, Bar-Zakai B, Hershko D, Shabtai M, Krausz MM, Ayalon A. Is mechanical bowel preparation mandatory for left-sided colonic anastomosis? Results of a prospective randomized trial. Tech Coloproctol. 2006;10(2):131-135. doi: 10.1007/s10151006-0266-1.

11. Güenaga KF, Matos D, Wille-Jørgensen P. Mechanical bowel preparation for elective colorectal surgery. Cochrane Database Syst Rev. 2011;2011(9):CD001544. doi: 10.1002/14651858.CD001544.pub4.

12. Slim K, Vicaut E, Launay-Savary MV, Contant C, Chipponi J. Updated systematic review and meta-analysis of randomized clinical trials on the role of mechanical bowel preparation before colorectal surgery. Ann Surg. 2009;249(2):203-209. doi: 10.1097/ SLA.0b013e318193425a.

13. Clarke JS, Condon RE, Bartlett JG, Gorbach SL, Nichols RL, Ochi S. Preoperative oral antibiotics reduce septic complications of colon operations: results of prospective, randomized, double-blind clinical study. Ann Surg. 1977;186(3):251-259. doi: 10.1097/00000658197709000-00003.

14. Scarborough JE, Mantyh CR, Sun Z, Migaly J. Combined Mechanical and Oral Antibiotic Bowel Preparation Reduces Incisional Surgical Site Infection and Anastomotic Leak Rates After Elective Colorectal Resection: An Anal- ysis of Colectomy-Targeted ACS NSQIP. Ann Surg. 2015;262(2):331-337. doi: 10.1097/ SLA.0000000000001041.

15. Kiran RP, Murray AC, Chiuzan C, Estrada D, Forde K. Combined preoperative mechanical bowel preparation with oral antibiotics significantly reduces surgical site infection, anastomotic leak, and ileus after colorectal surgery. Ann Surg. 2015;262(3):416-425. doi: 10.1097/ SLA.0000000000001416.

16. Rollins $K E$, Javanmard-Emamghissi $H$, Acheson AG, Lobo DN. The role of oral antibiotic preparation in elective colorectal surgery: A meta-analysis. Ann Surg. 2019;270(1):43-58. doi: 10.1097/SLA.0000000000003145.

17. Toh JWT, Phan K, Hitos K, Pathma-Nathan N, El-Khoury T, Richardson AJ, Morgan G, Engel A, Ctercteko G. Association of mechanical bowel preparation and oral antibiotics before elective colorectal surgery with surgical site infection: A network meta-analysis. JAMA Netw Open. 2018;1(6):e183226. doi: 10.1001/ jamanetworkopen.2018.3226.

18.2017 European Society of Coloproctology (ESCP) collaborating group. Association of mechanical bowel preparation with oral antibiotics and anastomotic leak following left sided colorectal resection: an international, multi-centre, prospective audit. Colorectal Dis. 2018;20 Suppl 6:15-32. doi: 10.1111/ codi.14362.

19. Abis GSA, Stockmann HBAC, Bonjer HJ, van Veenendaal $\mathrm{N}$, van Doorn-Schepens MLM, Budding $A E$, Wilschut $J A$, van Egmond $M$, Oosterling SJ; SELECT trial study group. Randomized clinical trial of selective decontamination of the digestive tract in elective colorectal cancer surgery (SELECT trial). Br J Surg. 2019;106(4):355-363. doi: 10.1002/bjs.11117.

20. Schardey HM, Wirth U, Strauss T, Kasparek MS, Schneider D, Jauch KW. Prevention of anastomotic leak in rectal cancer surgery with local antibiotic decontamination: a prospective, randomized, double-blind, placebo-controlled single center trial. Int J Colorectal Dis. 2020;35(5): 847-857. doi: 10.1007/s00384-020-03544-8.

21. Koskenvuo L, Lehtonen T, Koskensalo S, Rasilainen S, Klintrup K, Ehrlich A, Pinta T, Schei- 
nin T, Sallinen V. Mechanical and oral antibiotic bowel preparation versus no bowel preparation for elective colectomy (MOBILE): a multicentre, randomised, parallel, single-blinded trial. Lancet. 2019;394(10201):840-848. doi: 10.1016/S0140-6736(19)31269-3.

22. Garfinkle R, Abou-Khalil J, Morin N, Ghitulescu G, Vasilevsky CA, Gordon P, Demian M, Boutros $M$. Is there a role for oral antibiotic preparation alone before colorectal surgery? ACS-NSQIP analysis by coarsened exact matching. Dis Colon Rectum. 2017;60(7):729737. doi: $10.1097 / D C R .0000000000000851$.
23. Migaly J, Bafford AC, Francone TD, Gaertner WB, Eskicioglu C, Bordeianou L, Feingold DL, Steele SR; Clinical Practice Guidelines Committee of the American Society of Colon and Rectal Surgeons. The American Society of Colon and Rectal Surgeons Clinical Practice Guidelines for the Use of Bowel Preparation in Elective Colon and Rectal Surgery. Dis Colon Rectum. 2019;62(1):3-8. doi: 10.1097/ DCR.0000000000001238.

24. Vogel JD, Eskicioglu C, Weiser MR, Feingold DL, Steele SR. The American Society of Colon and Rectal Surgeons Clinical Practice Guidelines for the Treatment of Colon Cancer. Dis Colon Rectum. 2017;60(10):999-1017. doi: 10.1097/ DCR.0000000000000926.

25. Holubar SD, Hedrick T, Gupta R, Kellum J, Hamilton M, Gan TJ, Mythen MG, Shaw AD, Miller TE; Perioperative Quality Initiative (POQI) I Workgroup. American Society for Enhanced Recovery (ASER) and Perioperative Quality Initiative (POQI) joint consensus statement on prevention of postoperative infection within an enhanced recovery pathway for elective colorectal surgery. Perioper Med (Lond). 2017;6:4. doi: 10.1186/s13741-017-0059-2.

\title{
Preoperative mechanical bowel preparation combined with oral antibacterials in the prevention of complications of surgery for rectal and rectosigmoid junction cancer
}

\author{
Yu.V. Ivanov ${ }^{1,2}$ • A.V. Smirnov' • A.V. Vinokurov' • A.I. Zlobin' • \\ V.R. Stankevich ${ }^{1}$ E.S. Danilina'
}

Aim: To evaluate the efficacy of mechanical bowel preparation (MBP) combined with oral antibacterials for the prevention of postoperative complications when preparing a patient for anterior rectal resection.

Materials and methods: We analyzed shortterm results in 77 patients who had undergone anterior rectal resection for rectal and rectosigmoid junction cancer. Forty five (45) patients were prepared for surgery only with MBP. In 32 patients, in addition to MBP, oral antibacterial agents ciprofloxacin and metronidazole were used preoperatively.

Results: The overall rate of postoperative complications was $6.25 \%$ (2/32 patients) in the group of combined preparation for surgery and $15.5 \%(7 / 45)$ in the group using only MBP. Surgical wound infection occurred in 1 patient in the combined preparation group and in 4 patients in the MBP only group. There was no anastomotic leak in the combined preparation group, whereas in the MBP only group, anastomotic leak occurred in 2 patients.
Conclusion: Combined use of oral antibacterials and MBP before anterior rectal resection makes it possible to achieve an extremely low rate of the colorectal anastomosis leak. Further studies into the efficacy of this preparation regimen are needed, along with their discussion in the professional communities.

Key words: colorectal cancer, anterior rectal resection, intestinal microflora, colorectal anastomosis incompetence

For citation: Ivanov YuV, Smirnov AV, Vinokurov AV, Zlobin Al, Stankevich VR, Danilina ES. Preoperative mechanical bowel preparation combined with oral antibacterials in the prevention of complications of surgery for rectal and rectosigmoid junction cancer. Almanac of Clinical Medicine. 2021;49(7):477-484. doi: 10.18786/2072-0505-2021-49-062.

Received 6 September 2021; revised 11 December 2021; accepted 13 December 2021; published online 16 December 2021

\section{Funding}

The study was performed as a part of the State Project of the Federal Medical and Biological Agency \# 121031900022-7. Authors' contributions

Yu.V. Ivanov, concept and design of the study, text writing and editing; A.V. Smirnov, concept and design of the study, data analysis, text writing and editing; A.V. Vinokurov, V.R. Stankevich and E.S. Danilina, data collection and management; A.I. Zlobin, statistical analysis, literature search and analysis. All the authors have read and approved the final version of the manuscript before submission, agreed to be accountable for all aspects of the work in ensuring that questions related to the accuracy or integrity of any part of the work are appropriately investigated and resolved.

Yuri V. Ivanov - MD, PhD, Professor, Head of Department of Surgery'; Chief Research Fellow, Laboratory of Minimally Invasive Surgery2 ${ }^{2}$ ORCID: https://orcid.org/0000-0001-6209-4194.

E-mail: ivanovkb83@yandex.ru

Alexander V. Smirnov - MD, PhD, Surgeon, Department of Surgery'; ORCID: https://orcid. org/0000-0003-3897-8306

$\square 28$ Orekhovyy bul'var, Moscow, 115682, Russian Federation. Tel.: +7 (926) 2048148.

E-mail: alvsmirnov@mail.ru

Aleksandr V. Vinokurov - Resident, Chair of Surgery, Academy of Postgraduate Education'. ORCID: https://orcid.org/0000-0001-8026-1216. E-mail: vinokuroff.sasha2015@yandex.ru

Alexander I. Zlobin - MD, PhD, Surgeon, Department of Surgery ${ }^{1}$

Vladimir R. Stankevich - Surgeon, Department of Surgery'; ORCID: https://orcid.org/0000-0002-86208755. E-mail: v-stankevich@yandex.ru

Ekaterina S. Danilina - Surgeon, Department of Surgery; Senior Laboratory Assistant, Chair of Surgery, Academy of Postgraduate Education'; ORCID: https://orcid.org/0000-0002-2466-3795. E-mail: danilina.katja@bk.ru

Federal Scientific and Clinical Center for Specialized Types of Medical Care and Medical Technologies; 28 Orekhovyy bul'var, Moscow, 115682, Russian Federation

${ }^{2}$ A.I. Evdokimov Moscow State University of Medicine and Dentistry; 20-1 Delegatskaya ul., Moscow, 127473, Russian Federation 